\title{
Mechanical Analysis of Chorion Softening in Prehatching Stages of Zebrafish Embryos
}

\author{
Deok-Ho Kim, Member, IEEE, Chang Nam Hwang, Yu Sun, Member, IEEE, Sang Ho Lee, Byungkyu Kim*, and \\ Bradley J. Nelson, Member, IEEE
}

\begin{abstract}
During early development, the chorion envelope of the zebrafish embryo undergoes a thinning process called "chorion softening," which has so far only been characterized chemically. In this study, a micromechanical force sensing system was used to characterize and quantitate mechanical modifications of the zebrafish embryo chorion during early development. Quantitative relationships between applied forces and chorion structural deformations were established at various embryonic stages. The measured penetration force into the chorion at the blastula stage was 1.3-fold greater than those at the prehatching stage. Furthermore, chorion elastic modulus values were determined by using a biomembrane elastic model. The elastic modulus of the chorion at the blastula stage was 1.66 -fold greater than that at the prehatching stage, thus indicating that the chorion envelope become mechanically "softened" at the prehatching stage. The experimental results quantitatively describe "chorion softening," which is most likely due to proteolytic activities at the prehatching stage. Gradual chorion softening during embryonic development was also artificially achieved by treating blastula chorion with pronase, a proteolytic enzyme. The forces required to penetrate the pronase-treated chorion were similar to those at the prehatching stage. This similarity suggests that "chorion softening" may be induced by the release of protease from the embryos, and the chemical nature of the process involves proteolytic fragmentation of the ZP2 protein.
\end{abstract}

Index Terms-Cellular force sensing, chorion softening, elastic modulus, hatching, peptide fragmentation, protease, proteolytic activities, zebrafish embryo.

\section{INTRODUCTION}

$\mathbf{T}$ HE relationship between mechanical forces and cellular deformations in living organisms is important for understanding a variety of cell functions. Several methods have been

Manuscript received July 20, 2005; revised November 8, 2005. This work was supported by the 21st Century's Frontier R\&D Projects, under Contract MS-02-324-01, sponsored by the Ministry of Science and Technology, Korea, in part by the Schweizerische Nationalfonds zur Förderung der wissenschaftlichen Forschung, and in part by the U.S. National Science Foundation under Grant IIS-0208564. The first two authors contributed equally to this work. Asterisk indicates corresponding author.

D.-H. Kim is with the Microsystem Research Center, Korea Institute of Science and Technology, Seoul 138-791, Korea (e-mail: dhkim21c@gmail.com).

C. N. Hwang and S. H. Lee are with the School of Life Sciences and Biotechnology, Korea University, Seoul 136-701, Korea (e-mail: sangho@ korea.ac.kr).

Y. Sun is with the Department of Mechanical and Industrial Engineering and the Institute of Biomaterials and Biomedical Engineering, University of Toronto, Toronto, ON M5S 3G8, Canada (e-mail: sun@mie.utoronto.ca).

*B. Kim is with the School of Aerospace and Mechanical Engineering, Hankuk Aviation University, Kyonggi-do 412-791, Korea (e-mail: bkim@hau.ac.kr).

B. J. Nelson is with the Swiss Federal Institute of Technology (ETH-Zurich), CH-8092 Zurich, Switzerland (e-mail: bnelson@ethz.ch).

Digital Object Identifier 10.1109/TNB.2006.875054 developed for mechanically characterizing cell membranes including the extracellular matrix (ECM) [1]-[7]. A tensile test system has been designed and applied to fibroblasts from the rabbit patellar tendon [1]. An effective instrument embodying a video enhanced microscope and a theoretical linear elastic solution were developed to characterize the elastic modulus of a thin biological membrane [2]-[4]. The mechanical behavior of living cells was studied with micropipette suction in which the surface of a cell is aspirated into a capillary pipette while tracking the leading edge of its surface [5]. Microelectromechanical systems (MEMS)-based cellular force sensors were developed to quantitate mechanical property changes of the mouse zona pellucida (ZP) during fertilization [6] or measure forces generated by living heart muscle cells [7]. Despite these efforts, there has been no quantitative study on the chorion, which is the ECM of zebrafish embryos.

The zebrafish is a recently emerging model organism for addressing questions of vertebrate embryo development [8]-[10]. The chorion envelope of zebrafish embryos undergoes a thinning process called "chorion softening" before the basic body formation is complete and hatching occurs [11]. Upon hatching, the chorion is digested by hatching enzymes that are proteolytic enzymes secreted from hatching gland cells of the embryo [12], [13]. The hatching gland cells of zebrafish are in the epidermis of the yolk sac and are originated from the anterior end of the hypoblast, the polster [14]. The hatching enzymes have been partially identified, which may be responsible for "chorion softening" [15].

Similar to chemically characterizing "zona hardening" [16]-[18], biological studies have employed chemicals to test the period for dissolving the chorion, which chemically rather than mechanically describes hardness/softness of the chorion. For example, proteases, such as high choriolytic enzyme (HCE) and cathepsin L, that are a constituent protease of the hatching enzyme were shown to bind tightly to the chorion as well as swell the chorion by partially hydrolyzing it [8], [19]. The chorion envelope undergoes chemical modifications during both fertilization and hatching for successful embryonic development. It was speculated that these chemical modifications might cause mechanical changes.

The zebrafish is an attractive model for manipulations and studies of biophysical properties due to the relatively large egg size and the fact that basic cellular processes (i.e., fertilization, embryonic development, and hatching) involving the ECM are well-conserved among vertebrates. Therefore, an understanding of mechanical modifications of zebrafish egg ECM will allow for obtaining insights into the clinical problems arising in 


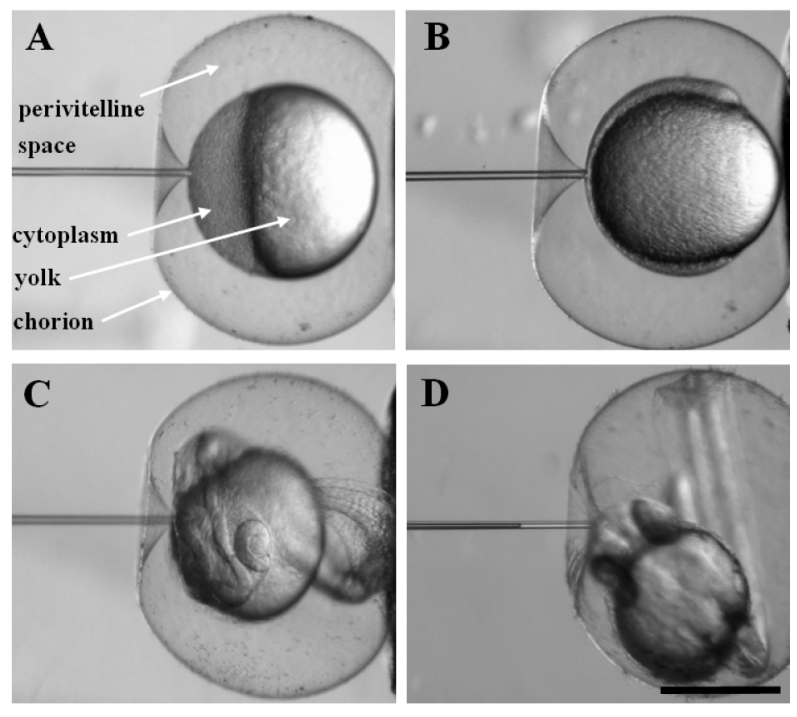

Fig. 1. Zebrafish embryos at different developmental stages. Pipette angle in the loading experiments is carefully controlled so that the pipette is loaded perpendicularly to the chorion. Blastula (A, $3 \mathrm{hpf}$ ); gastrula (B, $7.5 \mathrm{hpf})$; pharyngula (C, $24 \mathrm{hpf}$ ); and prehatching stage (D, $36 \mathrm{hpf}$ ). Scale bar is $200 \mu \mathrm{m}$.

polyspermy and implantation failure due to inappropriate ECM hardening and incomplete hatching. For example, one of the zebrafish chorion components called ZPC protein gene has been cloned and identified as a homologous zebrafish gene product to a mouse ZP protein, called ZP3. Upon fertilization, the zebrafish chorion undergoes chemical modifications similar to mouse ZP proteins during fertilization [20].

In order to quantitate the mechanical modifications of the chorion during early development, a micromechanical force sensing system was developed in this study. Quantitative relationships between applied forces and chorion structural deformations were established for various developmental stages. The gradual decrease of the measured chorion penetration forces from blastula to pharyngula stages was closely associated with a well-developed hatching gland that is present at the prehatching stage on the pericardium over the anterior yolk sac, secreting proteases to dissolve the chorion at the end of the embryonic development [21]. The chemical nature of the softening process may be due to the fragmentation of the ZP2 protein that is caused by the proteolytic enzymes released from hatching embryos.

\section{METHODS}

\section{A. Embryo Preparation}

For force-deformation measurements in developing embryos, ten embryos were collected from each typical developmental stage (Fig. 1). Embryos were accurately staged by examining morphological features of each living embryo under the microscope according to the standard stages of embryonic development of the zebrafish [21]. Each stage was clearly discriminated due to the transparent cell structure. Following the blastula stage, embryos develop into the gastrula stage at $7.5 \mathrm{~h}$ postfertilization (hpf) with three germ layers formed. The embryo, called pharyngula (prim-5) at $26 \mathrm{hpf}$, shows distinct movement within the chorion. Between 42 and $48 \mathrm{hpf}$, the stage is known as prehatching (high-pec). The embryo hatches

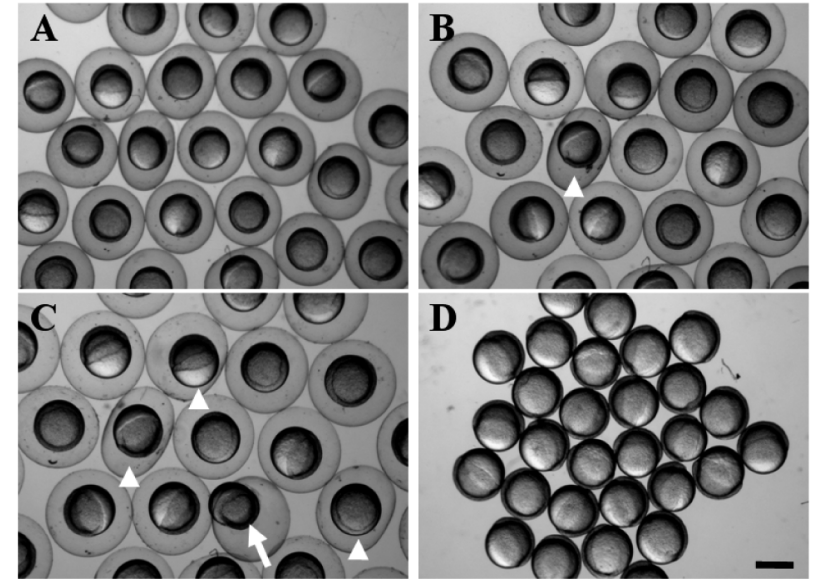

Fig. 2. Pronase induced artificial "chorion softening." embryos at the blastula stage were incubated for (a) 0 , (b) 10 , (c) 15, and (d) $40 \mathrm{~min}$ in pronase-containing ZCM. Note that the chorions of some embryos are swelling [arrowheads in (b) and (c)], hatching [an arrow in (c)] or denuded (d) due to the proteolytic enzyme. Scale bar is $300 \mu \mathrm{m}$.

at $48 \mathrm{hpf}$ by dissolving the chorion envelope with secreted protease enzymes. During protease secretion, the chorion becomes thinner, eventually forming a hole that is enlarged by the developing fry's movement.

Blastula embryos are more uniform in morphology compared to embryos at the gastrula stage when asynchronous development becomes evident. To examine penetration forces into lyzed embryos, ten embryos were lyzed by heating at the blastula stage in a petridish at a temperature of $40{ }^{\circ} \mathrm{C}$ for $20 \mathrm{~min}$. The embryos were subsequently used in force measurements after being placed at room temperature $\left(22^{\circ} \mathrm{C}-24{ }^{\circ} \mathrm{C}\right)$ for $2 \mathrm{~h}$.

Besides quantitating mechanical property differences of zebrafish chorion at various developmental stages, another purpose of this study is to investigate the effect of pronase treatment on the chorion mechanical property modifications.

Embryos used for the pronase treatment experiments were treated with 2 units pronase/ml (Type XIV, Sigma Chem. Co) without shaking the embryos in zebrafish culture medium (ZCM) at the blastula stage at $3 \mathrm{hpf}$. At appropriate intervals, the treated embryos were washed with $10 \%$ FBS in ZCM three times to remove the remaining pronase for $5 \mathrm{~min}$ each. The chorion was completely dissolved by pronase treatment for 40 min [Fig. 2(d)]. Before force measurements were conducted, four embryo groups were incubated for 1, 3, 5, and $7 \mathrm{~min}$ in pronase-containing ZCM, respectively.

\section{B. Micromechanical Force Sensing System}

The system consists of two micromanipulators (Fig. 3(e), model: MP-285, Sutter Inc.; Fig. 3(f), model: InjectMan NI2, Eppendorf) equipped with a holding pipette [Fig. 3(b)] and an injection pipette [Fig. 3(c)] on a vibration isolation table [Fig. 3(h)]. A precision $x-y$ translation stage (Fig. 3(g), model: M-410DG, PI Inc., Germany) was used to produce planar motion with a large workspace under a stereomicroscope (Fig. 3(i), model: MZ-12.5, Leica Inc., Germany). The images of zebrafish embryo chorion deformations during pipette loading were captured in real time $(30 \mathrm{~Hz})$. A polyvinylidene fluoride (PVDF) piezoelectric force sensor [Fig. 3(d)] with a 


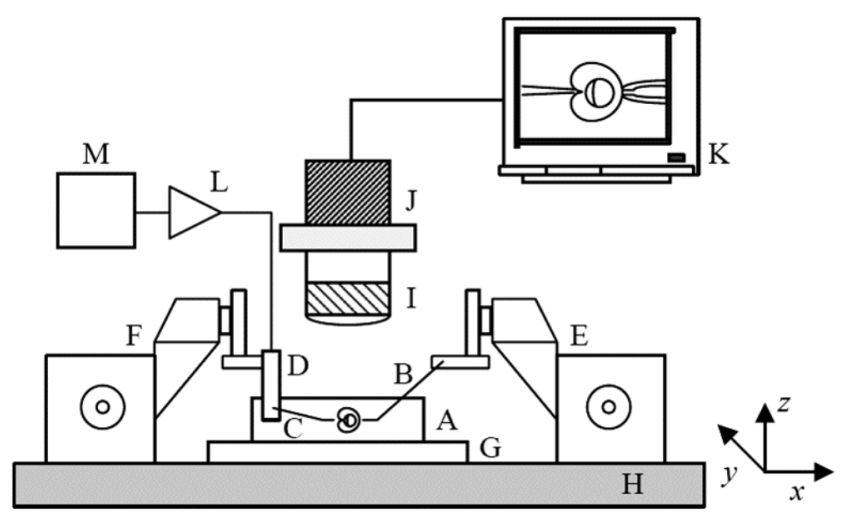

Fig. 3. A schematic of the micromechanical force sensing system for loading the chorion and measuring forces and chorion structural deformations. (a) Zebrafish embryo sample, (b) holding pipette, (c) injection pipette, (d) force transducer, (e and $\mathrm{f}$ ) micromanipulator, (g) precision $\mathrm{X}-\mathrm{Y}$ translation stage, (h) anti-vibration table, (i) microscope objective, (j) CCD camera, (k) image analysis system, (1) charge amplifier, and (m) data acquisition system are depicted on the scheme, respectively.

14.5- $\mu \mathrm{N}$ resolution [22] was used to detect chorion penetration forces. The injection pipette was bonded to the tip of the PVDF force sensor that was clamped on a fixture on the micromanipulator [Fig. 3(f)]. Charges generated by the PVDF sensor were amplified by a charge amplifier [Fig. 3(1)]. The output was sampled through a data acquisition board (Fig. 3(m), model: dSPACE 1103), after which signals were digitized and filtered for noise rejection. The end tip of the injection pipette was $14.6 \mu \mathrm{m}$ in radius. The pipette motion was controlled at a speed of $120 \mu \mathrm{m} / \mathrm{s}$.

\section{Protein Gel Electrophoresis}

To determine the chemical nature of "chorion softening" during prehatching stages, the chorions recovered from blastula embryos were partially digested for 10 min with 2 units pronase/ml to imitate the natural proteolytic activity caused by the embryo. The digested chorion solution along with nondigested chorion solution was collected and analyzed by sodium dodecylsulfate polyacrylamide gel electrophoresis (SDS-PAGE) [23] to compare the peptides before and after partial digestion. The gels were stained with $0.025 \%$ Coomasie Brilliant Blue.

\section{RESULTS AND DISCUSSION}

Mechanical property changes of the chorion were found both during fertilization and at the prehatching stage. The first experiment was conducted on developing zebrafish embryos without pronase treatment. The chorion loading experiments were performed at room temperature $\left(22-24^{\circ} \mathrm{C}\right)$. The force sensing profile and chorion structural deformations were obtained in real time during pipette penetration into the chorion envelope in blastula embryos. As shown in Fig. 4, the measured forces increased greatly during controlled chorion loading at a speed of $120 \mu \mathrm{m} / \mathrm{s}$. When deformation reaches approximately $170 \mu \mathrm{m}$, the chorion envelopes at the blastula stage were punctured, resulting in the maximum penetration forces.

For embryos at the blastula stage, a mean penetration force of $737 \mu \mathrm{N}$ was measured, after which the force signal dropped to the basal level (Fig. 4).

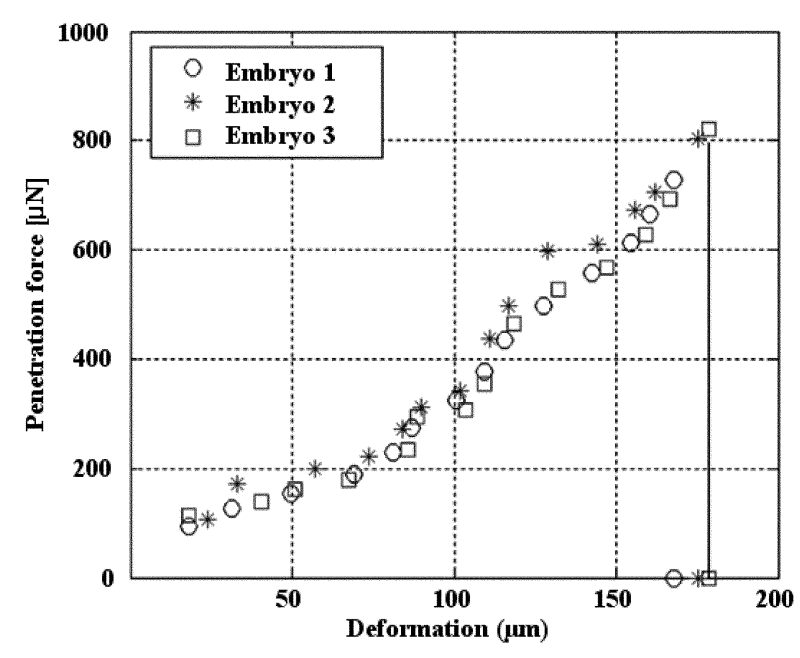

Fig. 4. Force-deformation curves of the zebrafish embryo chorion envelopes at the blastula stage. Experimental results of three embryos out of ten are shown. When deformation reached approximately $170 \mu \mathrm{m}$, the chorion envelopes were punctured, which resulted in the maximum forces measured (penetration forces).

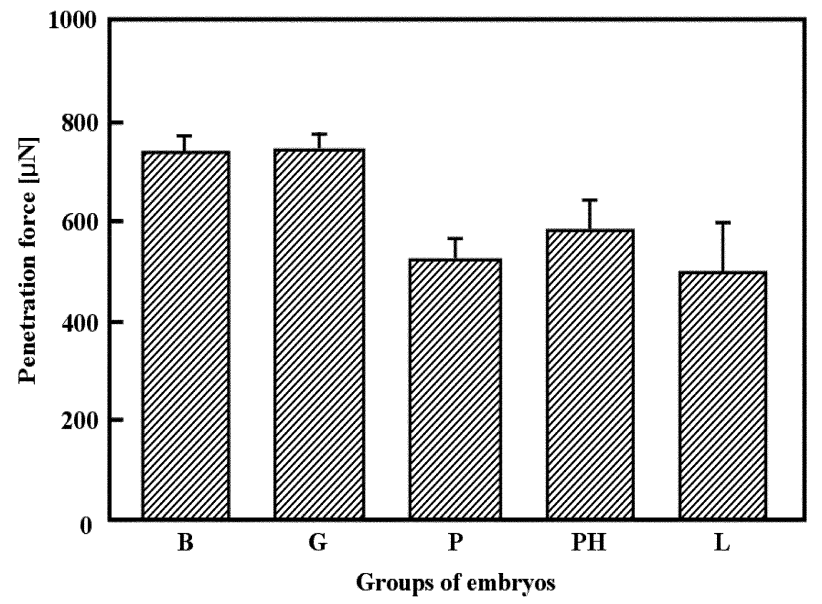

Fig. 5. Penetration force comparisons of the zebrafish embryo chorion at different developmental stages. Bars indicate values of measured average forces. Blastula (B), gastrula (G), pharyngula (P), prehatching $(\mathrm{PH})$, and lyzed embryos (L) were used in each experiment. The data were obtained from the measurements in ten embryos in each group. To prepare the lyzed embryos with disorganized cytoplasm, blastula embryos were used because blastula embryos are more uniform in morphology compared to embryos at the gastrula stage when asynchronous development becomes evident such that the protease secretion effect of lyzed embryos can be more obviously revealed.

Force-deformation curves of embryos at other developmental stages produced similar patterns although measured penetration forces slightly varied (Fig. 5). The required average forces to penetrate chorion envelopes at the blastula stage $(737 \mu \mathrm{N}$ with a standard deviation of $32.1 \mu \mathrm{N}(1 \delta))$ and gastrula stage $(738 \mu \mathrm{N}$ with a standard deviation of $35.1 \mu \mathrm{N}(1 \delta)$ ) are 1.3-fold greater than those at the prehatching stage $(578 \mu \mathrm{N}$ with a standard deviation of $63 \mu \mathrm{N}(1 \delta)$ ).

In order to estimate elastic modulus value changes of the zebrafish chorion at different developmental stages, a cell membrane mechanics model was used [6]. A deformed embryo membrane was approximated by a local dimple and a toroidal surface. Parameter values for the indentation of a zebrafish embryo 


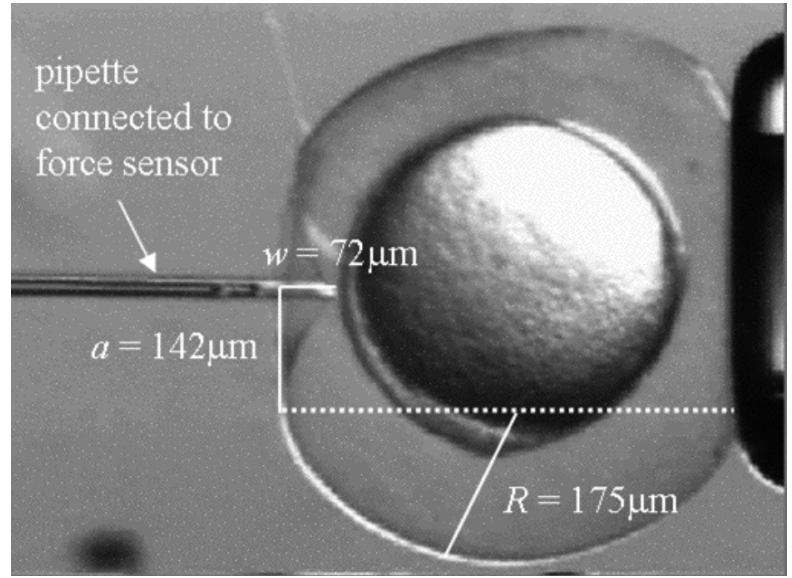

Fig. 6. Chorion loading and penetration. The pipette on the left is connected to the PVDF force sensor for force measurements. On the right is a holding pipette for fixing the cell. The geometrical parameters values are shown for the deformed chorion.

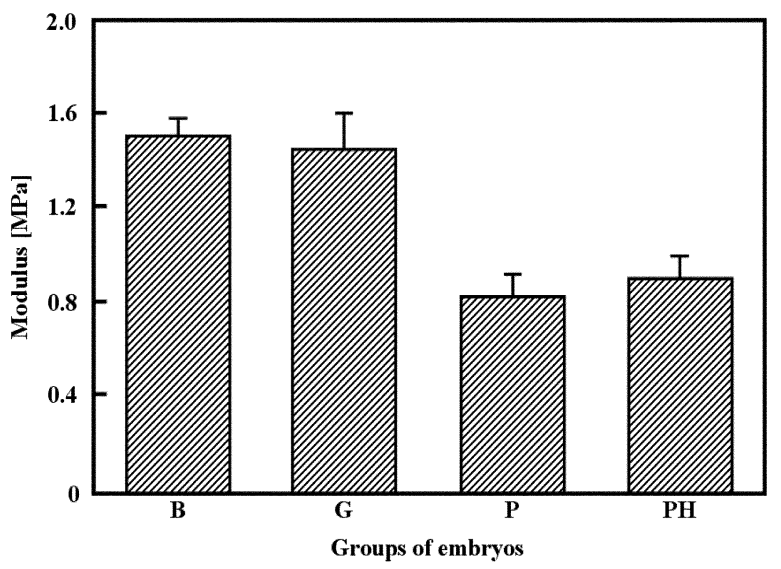

Fig. 7. Estimated elastic modulus values of the zebrafish embryo chorion at different developmental stages. Blastula $(B)$, gastrula $(\mathrm{G})$, pharyngula $(\mathrm{P})$, and prehatching embryos $(\mathrm{PH})$ were used in each experiment. The data were obtained from the measurements in ten embryos in each group.

by a pipette (cylindrical indenter) (Fig. 6) were determined in order to calculate elastic modulus values using

$$
F=\frac{2 \pi E h w^{3}}{a^{2}(1-\nu)}\left[\frac{3-4 \varsigma^{2}+\varsigma^{4}+2 \ln \varsigma^{2}}{\left(1-\varsigma^{2}\right)\left(1-\varsigma^{2}+\ln \varsigma^{2}\right)^{3}}\right]
$$

where $\varsigma=c / a$. The geometric parameters $a$ and $w$ were measured from captured images. $F$ is the measured force. The poisson ratio $\nu$ was assumed to be 0.5 . The constants are $3 \mu \mathrm{m}$ for chorion's thickness $(h)$ and $14.6 \mu \mathrm{m}$ for indenter radius $(c)$. Elastic modulus $(E)$ based on 14 data points from embryos at the blastula and gastrula stages and nine data points from embryos at the pharyngula and prehatching stages were thus calculated. Throughout pipette loading, the modulus values were found to be fairly constant (Fig. 7). Elastic modulus values for individual chorion envelopes varied only slightly from the mean values. The mean elastic modulus value of the chorion envelopes at the blastula stage (1.51 MPa with a standard deviation of $0.07 \mathrm{MPa}(1 \delta)$ ) was 1.66 -fold greater than that at

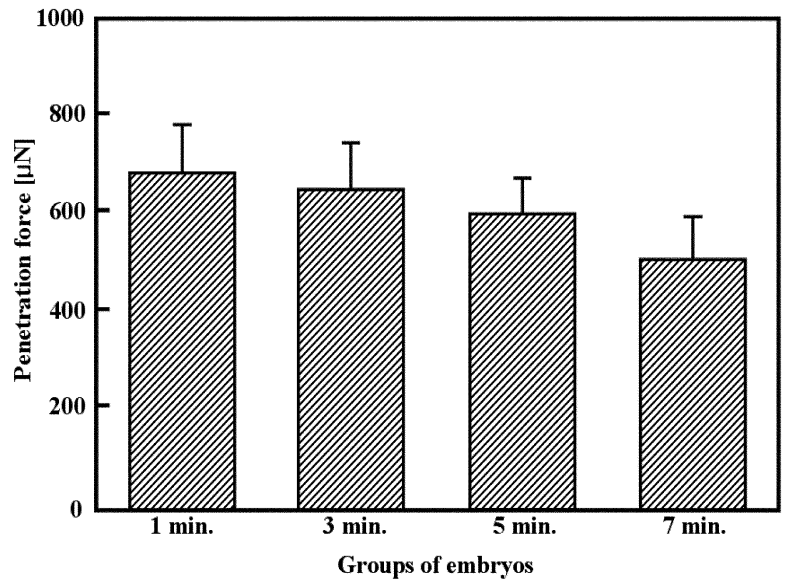

Fig. 8. Penetration force comparisons in the chorion envelopes of zebrafish embryos treated with pronase. Artificial "chorion softening" was achieved by pronase treatment. The embryos were treated with pronase for 1, 3, 5, and $7 \mathrm{~min}$ in the respective groups. The data were obtained from the measurements from ten embryos each.

the prehatching stage $(0.91 \mathrm{MPa}$ with a standard deviation of $0.09 \mathrm{MPa}(1 \delta))$.

The second experiment was conducted to determine the mechanical properties of the lyzed zebrafish embryo chorion. Mean penetration forces of $500 \mu \mathrm{N}$ with a standard deviation of $100 \mu \mathrm{N}(1 \delta)$ were measured in ten lyzed embryos when the chorion envelopes were subjected to cell lysis of the embryos. It is known that lyzed embryos release cellular hydrolase including protease [14], [24]. Lysed embryos are similar to the embryos at the prehatching stage in the sense that the chorion envelopes are both affected by protease activities by normal protease secretion (prehatching) and abrupt release of protease from dying cells (lyzed).

The third experiment was conducted to determine the effect of pronase treatment on mechanical properties of the zebrafish embryo chorion. Four groups of embryos were used in the experiment, each consisting of ten embryos. Each group was treated by pronase for $1,3,5$, and $7 \mathrm{~min}$, respectively. Note that the chorion envelopes of some embryos were swelling [arrows in Fig. 2(b) and Fig. 2(c)] or hatching [an arrow in Fig. 2(c) and all embryos in Fig. 2(d)] due to the action of the protelytic enzyme, pronase. The chorion penetration forces of pronase-treated embryos revealed that lower forces were required for penetrating the pronase-treated chorion in a time-dependent manner (Fig. 8), which demonstrates that pronase treatment is capable of inducing an artificial "chorion softening" effect.

Finally, the electrophoretic analysis of the softened chorion by partial chorion digestion with pronase showed that fragments of ZP2 protein are present (Fig. 9), which was verified by a MALDI-TOF analysis of the protein fragments separated by gel electrophoresis (unpublished data). Therefore, it is predicted that as the hatching stage approaches, the proteolytic enzymes released from the embryo soften the chorion by degrading the major protein ZP2 into several peptide fragments. The other minor unknown proteins were not detected among the visible peptide bands in the separated gel of the partially digested chorion solution when stained with Coomasie blue. Therefore, 


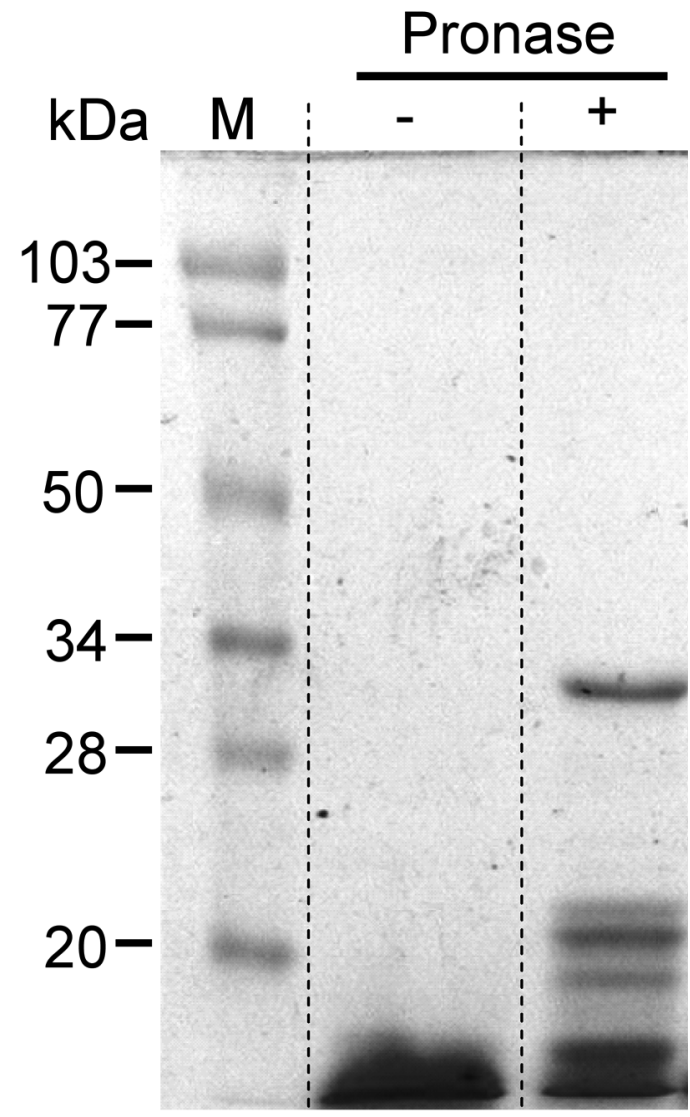

Fig. 9. Fragmentation of ZP2 protein in partially digested zebrafish chorion. To determine the chemical nature of "chorion softening" during prehatching stages, the chorions were treated with pronase for $10 \mathrm{~min}$. The resulting chorion solutions without $(-)$ or with pronase digestion $(+)$ were separated by SDS-PAGE and stained with Coomasie Brilliant Blue to demonstrate peptide components present in the chorion solutions. M, molecular standard makers, and relative molecular masses (in kilo Daltons, $\mathrm{kDa}$ ) are indicated on the left. A single protein band is seen in the lane without pronase, which is probably due to the presence of extremely abundant carbohydrates (anion charges) in the chorion. On the other hand, several peptide bands, apparently proteolytic products of the chorion, are present in the lane with pronase. The analysis of the peptide bands indicate that all major bands are derived from the ZP2 protein (see the text).

it appears that the spontaneously moving embryo at prehatching stage [Fig. 1(d)] should be able to make an opening in the weakened chorion having $\mathrm{ZP} 2$ fragmented during hatching process.

Thus, "chorion softening" was quantitated for both prehatching embryos and pronase-treated embryos, and its chemical nature was determined as a fragmentation of the ZP2 protein. In the prehatching embryos, the lower penetration forces are most likely due to proteolytic activity. Interestingly, the gradual decrease of penetration forces in pronase-treated embryo in a time-dependent manner suggests that pronase modification produces effects similar to the effects due to the gradual increase of proteolytic activity in prehatching embryos. These are the first experimental evidences that mechanical property modifications of the chorion are due to the proteolytic activities, and that ZP2 fragmentation occurs during the alteration. The results quantitatively demonstrate that mechanical property differences during "chorion softening" are most likely due to proteolytic enzymes secreted by embryos. This chemical process involves the fragmentation of the ZP2 protein as confirmed by the protein gel electrophoresis results.

\section{CONCLUSION}

The reported results in this paper present a quantitative study of zebrafish chorion mechanical modifications during early development. Through the use of a micromechanical force sensing system and a biomembrane elastic model, penetration forces and chorion elastic modulus values at various developmental stages were determined. Decreases in penetration forces and elastic modulus values were found during zebrafish embryo development. "Chorion softening" is most likely caused by proteolytic activities at the prehatching stage. This study also revealed the effect of pronase treatment on zebrafish embryo chorion, which produces an artificial "chorion softening" effect. An analogy was drawn between pronase treatment and proteolytic activities at the prehatching stage. Quantitating mechanical modifications of the zebrafish embryo chorion at different developmental stages provides a better understanding of zebrafish embryo development.

\section{ACKNOWLEDGMENT}

The authors would like to thank S. Yun for his technical assistance in data acquisition.

\section{REFERENCES}

[1] H. Miyazaki, Y. Hasegawa, and K. Hayashi, "A newly designed tensile tester for cells and its application to fibroblasts," J. Biomech., vol. 33 , pp. 97-104, 2002.

[2] B. F. Ju, K. K. Lui, S.-F. Ling, and W. H. Ng, "A novel technique for characterizing elastic properties of thin biological membrane," Mech. Mater., vol. 34, pp. 749-754, 2002.

[3] Y. Murayama, C. E. Constantinou, and S. Omata, "Micromechanical sensing platform for the characterization of the elastic properties of the ovum via uniaxial measurement," J. Biomech., vol. 37, pp. 67-72, 2004.

[4] M. T. A. Saif, C. R. Sager, and S. Coyer, "Functionalized biomicroelectromechanical systems sensors for force response study at local adhesion sites of single living cells on substrates," Ann. Biomed. Eng., vol. 31, pp. 950-961, 2003.

[5] R. M. Hochmuch, "Micropipette aspiration of living cells," $J$. Biomech., vol. 33, pp. 15-22, 2002.

[6] Y. Sun, K. T. Wan, K. P. Roberts, J. C. Bischof, and B. J. Nelson, "Mechanical property characterization of the mouse zona pellucida," IEEE Trans. Nanobiosci., vol. 2, no. 4, pp. 279-286, Dec. 2003.

[7] G. Lin, K. S. Pister, and K. P. Roos, "Surface micromachined polysilicon heart cell force transducer," J. Microelectromech. Syst., vol. 9, pp. 9-17, 2000.

[8] K. Inohaya, S. Yasumasu, K. Araki, K. Naruse, K. Yamazaki, I. Yasumasu, I. Iuchi, and K. Yamagami, "Species-dependent migration of fish hatching gland cells that express astacin-like proteases in common," Dev. Growth Differ., vol. 39, pp. 191-197, 1997.

[9] D. Stainier, "Zebrafish genetics and vertebrate heart formation," Nature Rev. Genetics, vol. 2, pp. 39-48, 2001.

[10] K. Y. Lee, H. Huang, B. Ju, Z. Yang, and S. Lin, "Cloned zebrafish by nuclear transfer from long-term-cultured cells," Nature Biotechnol., vol. 20, pp. 795-799, 2002.

[11] A. F. Schoots, R. C. Meijer, and J. M. Denuce, "Dopaminergic regulation of hatching in fish embryos," Dev. Biol., vol. 100, pp. 59-63, 1983.

[12] J. Hiroi, K. Maruyama, K. Kawazu, T. Kaneko, R. Ohtani-Kaneko, and S. Yasumasu, "Structure and developmental expression of hatching enzyme genes of the Japanese eel Anguilla japonica: An aspect of the evolution of fish hatching enzyme gene," Dev. Genes Evol., vol. 214, pp. 176-184, 2004 
[13] M. Gonzalez-Doncel, M. Larrea, S. Sanchez-Fortun, and D. E. Hinton, "Influence of water hardening of the chorion on cadmium accumulation in medaka (Oryzias latipes) eggs," Chemosphere, vol. 52, pp. 75-83, 2003.

[14] K. Inohaya, S. Yasumasu, I. Yasumasu, I. Iuchi, and K. Yamagami, "Analysis of the origin and development of hatching gland cells by transplantation of the embryonic shield in the fish, Oryzias latipes," Dev. Growth Differ., vol. 41, pp. 557-566, 1999.

[15] B. C. Roberts and R. G. White, "Effects of angular wading on survival of trout eggs and pre-emergent fry," N. Amer. J. Fish. Manage., vol. 12, pp. 450-459, 1992.

[16] I. Demeestere, P. Barlow, and F. Leroy, "Hardening of zona pellucida of mouse oocytes and embryos in vivo and in vitro," Int. J. Fertil. Womens Med., vol. 42, pp. 219-222, 1997.

[17] L. L. Lindsay and J. L. Hedrick, "Proteolysis of Xenopus laevis egg envelope ZPA triggers envelope hardening," Biochem. Biophys. Res. Commun., vol. 324, pp. 648-654, 2004.

[18] K. Nayernia, I. M. Adham, R. Shamsadin, C. Muller, U. Sancken, and W. Engel, "Proacrosin-deficient mice and zona pellucida modifications in an experimental model of multifactorial infertility," Mol. Hum. Reprod., vol. 8, pp. 434-440, 2002.

[19] A. M. Vogel and T. Gerster, "Expression of a zebrafish cathepsin L gene in anterior mesendoderm and hatching gland," Dev. Genes Evol., vol. 206, pp. 477-479, 1997.

[20] L. D. Giacco, S. Diani, and F. Cotelli, "Identification and spatial distribution of the mRNA encoding an egg envelop component of the Cyprinid zebrafish, Danio rerio, homologous to the mammailian ZP3 (ZPC)," Dev. Genes Evol., vol. 210, pp. 41-46, 2000.

[21] C. B. Kimmel, W. W. Ballard, S. R. Kimmel, B. Ullmann, and T. F. Schilling, "Stages of embryonic development of the zebrafish," Dev. Dyn., vol. 203, pp. 253-310, 1995.

[22] D.-H. Kim, B. Kim, and H. Kang, "Development of a piezoelectric polymer-based sensorized microgripper for micromanipulation and microassembly," Microsyst. Technol., vol. 10, pp. 275-280, 2004.

[23] P. H. O'Farrell, "High resolution two-dimensional electrophoresis of proteins," J. Biol. Chem., vol. 250, pp. 4007-4021.

[24] H. W. Denker, "Structural dynamics and function of early embryonic coats," Cells Tissues Organs, vol. 166, pp. 180-207, 2000.

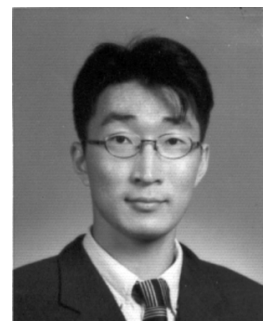

Deok-Ho Kim (M'04) received the B.S. degree in mechanical engineering from the Pohang University of Science and Technology (POSTECH), Pohang, Korea, in 1998 and the M.S. degree in mechanical design and production engineering from Seoul National University, Seoul, Korea, in 2000. $\mathrm{He}$ is currently working toward the Ph.D. degree in biomedical engineering at the Johns Hopkins University, Baltimore, MD.

$\mathrm{He}$ worked at Microsystem Research Center, Korea Institute of Science and Technology (KIST), Seoul, from 2000 to 2005. Between Nov. 2003 and Jun. 2004, he was a Visiting Research Scientist at the Swiss Federal Institute of Technology (ETH-Zurich). His research interests are in the areas of micro/nanorobotics, cellular bioMEMS, and cellular biomechanics.

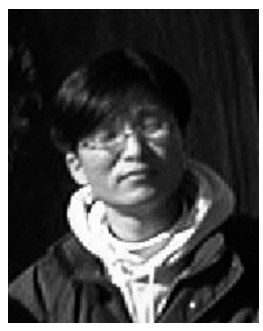

Chang Nam Hwang received the B.S. degree in marine biology from Kang Neung National University, Kangweon-do, Korea, in 1994, and the M.S. degree in reproductive biology from KunKuk University, Seoul, Korea, in 1996.He is currently working toward the Ph.D. degree in the Department of Biotechnology, Graduate School of Life Science and Biotechnology, Korea University, Seoul.

$\mathrm{He}$ is interested in the embryonic development of zebrafish, and molecular and cellular responses of stress in the developmental processes.

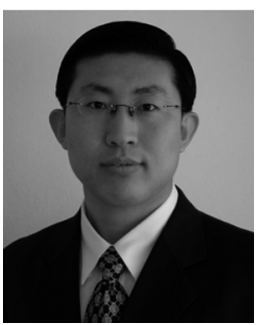

Yu Sun (S'01-M'03) received the B.S. degree in electrical engineering from the Dalian University of Technology, China, in 1996, the M.S. degree from the Institute of Automation, Chinese Academy of Sciences, Beijing, China, in 1999, and the Ph.D. degree in mechanical engineering from the University of Minnesota, Minneapolis, in 2003.

He held a Research Scientist position at the Swiss Federal Institute of Technology (ETH-Zurich) in 2003-2004. He is currently Assistant Professor of Mechanical and Industrial Engineering Department and is jointly appointed in the Institute of Biomaterials and Biomedical Engineering and the Department of Electrical and Computer Engineering at the University of Toronto, Toronto, ON, Canada. His research areas are bio-oriented micro and nanosystems, MEMS/NEMS design, fabrication and testing, control of microstructures, microrobotic biomanipulation and nanorobotics, biomechanics, and nanofabrication.

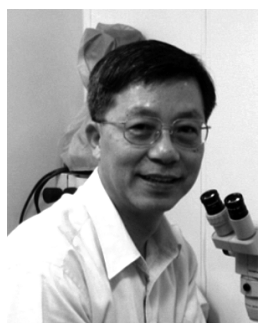

Sang Ho Lee received the B.S. and M.S. degrees from Korea University, Seoul, in reproductive biology, in 1978 and 1980, respectively, and the Ph.D. degree in developmental biology from University of London, London, U.K. in 1989.

He worked in the MRC Experimental Embryology and Teratology Unit, St. George's Hospital Medical School, London, as a visiting scientist and nonclinical research scientist for seven years. Currently, he is the Professor of Department of Molecular and Cellular Biology, Korea University. His main interests are molecular and cellular mechanisms involved in early embryonic development and stem cell researches for cell replacement therapy.

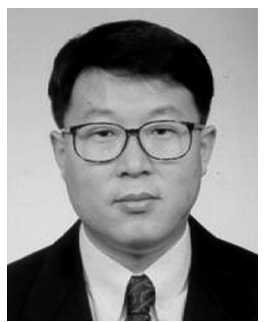

Byungkyu Kim received the Ph.D. degree in mechanical engineering from the University of Wisconsin, Madison, in 1997.

From 1997 to 2000, he was a Technical Staff Member of CXrL (Center for X-ray Lithography) at the University of Wisconsin, where he developed a computer code for thermal modeling of a mask membrane and wafer during beam exposure. $\mathrm{He}$ worked at the Microsystem Research Center, Korea Institute of Science and Technology, Seoul, Korea, from 2000 to 2005. He is currently an Assistant Professor in the School of Aerospace and Mechanical Engineering at the Hankuk Aviation University, Korea. His research interest includes microelectromechanical actuators, micro/nano manipulators for biomedical application, and microrobots.

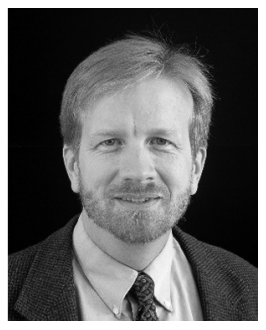

Bradley J. Nelson (M'90) received the B.S. degree in mechanical engineering from the University of Illinois, Urbana, in 1984, the M.S. degree in mechanical engineering from the University of Minnesota, Minneapolis, in 1987, and the Ph.D. degree in robotics from the School of Computer Science, Carnegie Mellon University, Pittsburgh, PA, in 1995.

$\mathrm{He}$ is currently the Professor of Robotics and Intelligent Systems at the Swiss Federal Institute of Technology (ETH), Zurich and heads the Institute of Robotics and Intelligent Systems there. His research interests are in the area of microrobotics, biomicrorobotics, and nanorobotics, including efforts in robotic micromanipulation, microassembly, MEMS (sensors and actuators), mechanical manipulation of biological cells and tissue, and NanoElectroMechanical Systems (NEMS). 